\title{
Automatisches Pollenmonitoring in Deutschland
}

\section{Eine Arbeit der Sektion Umwelt- und Arbeitsmedizin der Deutschen Gesellschaft für Allergologie und klinische Immunologie (DGAKI)}

Jeroen Buters ${ }^{1}$, Jose Oteros ${ }^{1}$, Robert Gebauer ${ }^{2}$, Katharina Heigl ${ }^{3}$

'ZAUM - Zentrum Allergie und Umwelt, Helmholtzzentrum München/Technische Universität München, ${ }^{2}$ IT Consulting Robert Gebauer, München, ${ }^{3}$ Bayrisches Landesamt für Gesundheit und Lebensmittelsicherheit (LGL), München

In Zeiten von Corona gibt es wichtigere Dinge als über Pollen nachzudenken. Jedoch beteiligt sich die Natur nicht an dem allgemeinen „Shutdown“: Der Pollenflug entwickelt sich unbeeinflusst weiter. Pollen können die Atemwege belasten und sind bestimmt nicht hilfreich, wenn gleichzeitig ein Virus die Atemwege angreift [1].

Deutschland ist weltweit Vorreiter auf dem Gebiet des automatischen Pollenmonitorings [2]. Initiator war das Bundesland Bayern, welches durch den Aufbau des elektronischen Polleninformationsnetzwerks (ePIN), bestehend aus acht automatischen Pollenmonitoren in Bayern, die Grundlagen für dieses neue System legte (Abb. 1) [3].

Die Daten der automatischen Pollenmonitore werden direkt ins Internet gestellt. Aktuelle Pollendaten sind abrufbar unter:

1. https://epin.bayern.de: Diese Website ist für interessierte Bürger und Allergiker gedacht und bietet Auskunft über den aktuellen Pollenflug der allergenen Pollen von Hasel, Erle, Birke, Esche sowie von Gräsern, Roggen, Beifuß und Ambrosia. Die Seite ist auf Deutsch, leicht verständlich und
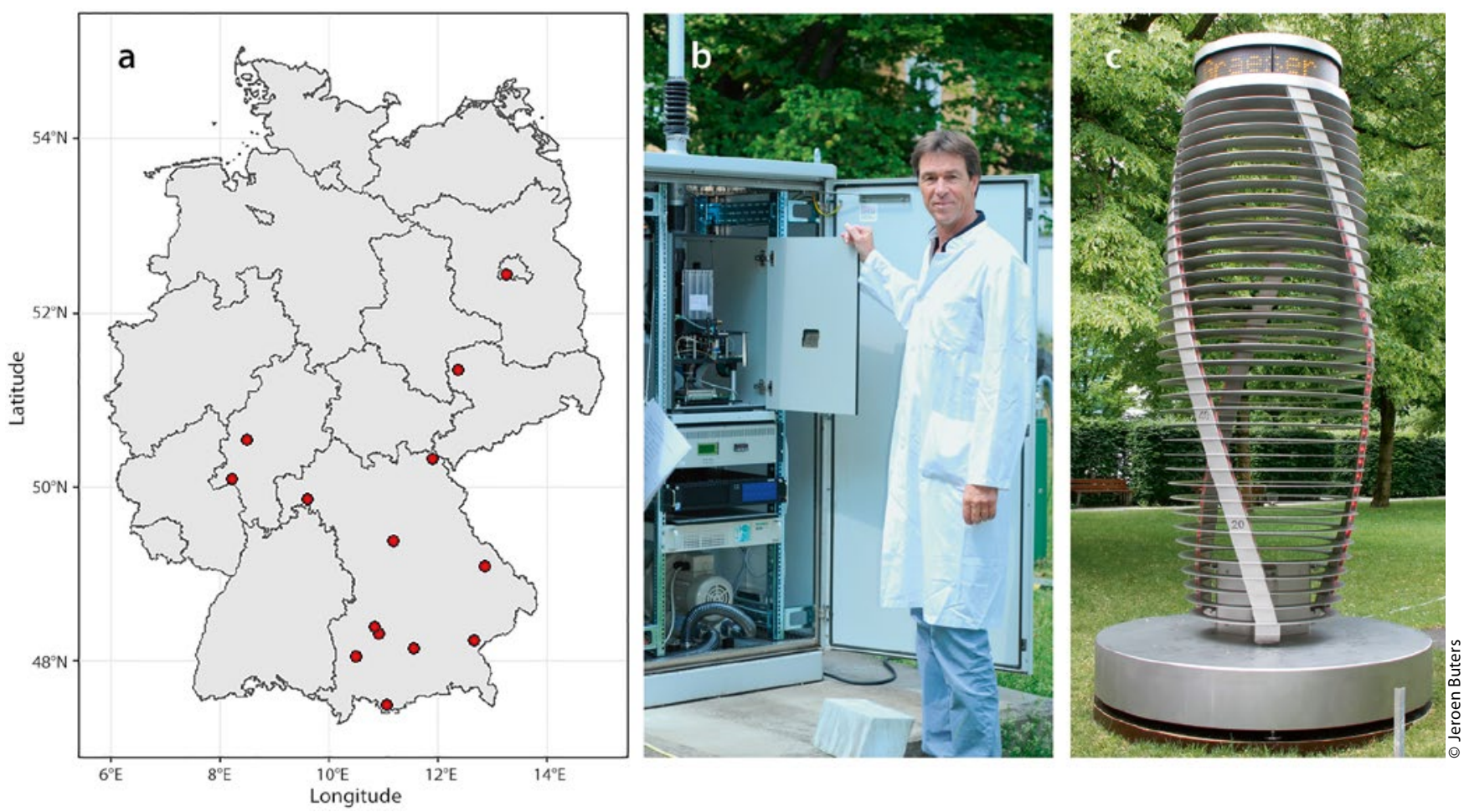

Abb. 1: Automatisches Pollenmonitoring in Deutschland. a: Karte mit Pollenmessstationen, die einen Pollenmonitor BAA500 betreiben. b: Ein Pollenmonitor mit Prof. Dr. Jeroen Buters, einem der Autoren. c: Freistehende Pollenampel (Kunstwerk, welches die aktuellen Pollenzahlen von München anzeigt; www.zaum-online.de/pollen/pollen-indicator.html) 
optisch ansprechend gestaltet; sie zeigt lediglich allergologisch relevante Pollenarten an den acht ePIN-Messstationen in Bayern an. Die Daten sind rückwirkend bis zum 15. Mai 2019 in Zwei-Wochen-Zeitfenstern aufrufbar.

2.https://epin-scientific.eu: Diese Webseite zeigt dieselben Daten wie „epin.bayern.de“, zusätzlich aber auch die Messstationen außerhalb Bayerns (Leipzig, Prof. Dr. Jan Simon; Berlin, Prof. Dr. Karl-Christian Bergmann; Wetzlar, Helmut Hund GmbH; Wiesbaden, Prof. Dr. Ludger Klimek; Payerne/ $\mathrm{CH}$, Dr. Bernard Clot). Auf dieser Seite werden alle gemessenen Pollenarten gezeigt, nicht nur allergene Pollenarten. Teilweise sind Pollendaten der letzten zehn Jahren zugänglich.

3. https://validation.epin.bayern: Diese Webseite enthält Detailinformationen. Das Bild von jedem gemessenen Pollen in ePIN-scientific ist hier abrufbar. Hier können Experten und auch Laien sehen, ob der Pollenmonitor einen Fehler gemacht hat (Benutzername: Gast; Passwort: ePIN-ZAUM). Experten können Fehler online korrigieren.

4. Freistehende Pollenampel (siehe Abb. 1c): Diese ist ein Kunstwerk von $4 \mathrm{~m}$ Höhe, das durch eine Lichtshow die aktuellen Pollenzahlen des Pollenmonitors in München anzeigt (www.zaum-online.de/pollen/pollen-indicator.html).

5.Zwei zusätzliche Pollenmonitore innerhalb Bayerns werden von Prof. Dr. Claudia Traidl-Hoffmann in Augsburg betrieben (https://wiki.unika-t.de/tiki-index.php?page=Pollenflug).

Die Webseite „epin.bayern.de“ sowie die zugehörige App werden vom Bayerischen Landesamt für Gesundheit und Lebensmittelsicherheit (LGL), dem Betreiber des elektronischen Polleninformationsnetzwerks (ePIN) in Bayern, zur Verfügung gestellt. ePIN ist Teil der bayerischen Klimaanpassungsstrategie und wird von den Bayerischen Staatsministerien für Gesundheit und Pflege (StMGP) sowie für Umwelt und Verbraucherschutz (StMUV) finanziert.

„epin-scientific.eu“ wird als wissenschaftliches Netzwerk betrieben. Neu in Betrieb genommene Pollenmonitore können dort problemlos aufgenommen werden. Voraussetzung dafür ist, dass als Messgerät der automatische Pollenmonitor BAA500 der Helmut Hund GmbH verwendet wird. Dies ist notwendig, da das System momentan auf dieses Gerät zugeschnitten ist und mit Bilderkennung zur Pollenidentifizierung arbeitet [4]. Der Betreiber dieser Website ist das ZAUM in München.

„validation.epin.bayern“ war ursprünglich ein wissenschaftliches Instrument, mit dem Pollenexperten die Pollenidentifizierung der Pollenmonitore kontrollierten. Die Identifizierung beinhaltet die Vergabe einer Pollenidentität durch die Software des Pollenmonitors oder manuell durch Pollenexperten. Die korrekte Identifizierung der Pollen war bislang nur durch den Hersteller selbst gesichert. Mit dieser Validierungssoftware haben Pollenexperten etwa 500.000 Pollenbilder betrachtet und die Identität zwischen dem manuellen (lichtmikroskopischen) und automatischen Ergebnis verglichen [5]. Der Betreiber dieser Website ist ebenfalls das ZAUM in München.

Der Roboter macht zwar Fehler, allerdings liegt die allgemeine korrekte Erkennungsrate bei etwa 91 \% [5]. Der Fehler des Roboters besteht meist darin, dass er einen Pollen nicht erkennt und als "unbekannt" einstuft. Ein Problem ist auch, dass

\section{Hier steht eine Anzeige.}

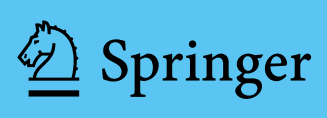


sich Experten untereinander bei der Bestimmung der Pollenidentität nicht immer einig sind [6]. Bei der Geschwindigkeit und Unermüdlichkeit ist der Roboter sicher unschlagbar - und wenn man an der Richtigkeit der Messungen des Roboters zweifelt, kann mit der Website „validation.epin.bayern“ jeder Pollen im Nachhinein analysiert werden.

Das automatische Pollenmonitoringssystem hat große Vorteile: Es ist online, es ist kontrollierbar und es liefert schnell Pollendaten. Sogar so schnell, dass ein Allergologe den Patienten fragen könnte: „Wie geht es Ihnen denn heute?“ Vom Patienten geäußerte allergische Symptome können dann umgehend mit dem lokalen Pollenflug in Beziehung gebracht werden. Handelt es sich um Covid-19 oder ist es vielleicht eine Allergie?

Der Großteil Deutschlands ist aber nicht ausreichend mit Pollenmonitoren abgedeckt. Dies hat unter anderem mit den Kosten zu tun. Bis heute werden die Pollenzahlen durch die Stiftung Deutscher Polleninformationsdienst aus manuellen Pollenfallen kostenlos geliefert; ein Service, der auf dem jahrzehntelangen, kostenlosen Einsatz von Prof. Dr. Bergmann und seinen Mitarbeitern beruht. Nun müsste die öffentliche Hand die Kosten übernehmen, wie es beispielsweise in Bayern schon geschehen ist.

Wie wird sich das automatische Pollenmonitoring in Deutschland weiterentwickeln? Klar ist, dass nie ausreichend Pollenmonitore aufgestellt werden können und dass dies sogar aus wissenschaftlicher Sicht nicht notwendig ist. Das Aufstellen von vielen Messpunkten ist zwar gut und wünschenswert, ein Simulationsmodel, das den genauen Pollenflug an jedem beliebigen Ort in einem Gebiet berechnet, ist aber die Zukunft [7]. Damit lassen sich nicht nur die Pollenzahlen beliebig genau lokal feststellen, ohne dass überall ein Messgerät steht, es können auch Vorhersagen für die Zukunft gemacht werden. Vorhersagemodelle, die konstant mit Daten aus automatischen Messstationen korrigiert werden, stellen zeit- und ortsgenaue Pollenzahlen am besten dar.

Bis es so weit ist, bräuchte man aber noch mehr automatische Pollenmonitore in Deutschland. Dabei muss sehr gut beachtet werden, dass man Pollen nicht an jedem beliebigen Ort messen kann und sollte. Die Frage „Wo ist es sinnvoll, einen Monitor aufzustellen?“ ist genauso wichtig wie „Welches Gerät sollen wir aufstellen?". Denn im Moment läuft ein Rennen zwischen konkurrierenden automatischen Pollenmessgeräten $[5,8,9]$. Die Frage des richtigen Ortes wird leider vernachlässigt, weil oft ältere Pollenmonitore durch neuere Generationen von Pollenmessgeräten am gleichen Ort ersetzt werden. Ob der Ort, an dem gemessen wurde, überhaupt geeignet ist, sollte jedoch vorher berücksichtigt werden.

Automatische Pollenmonitore sind bereits vorhanden, funktionieren zuverlässig und genau und werden ständig verbessert. Insbesondere die künstliche Intelligenz macht Fortschritte bei der Bilderkennung und wird zu noch besseren Wiedererkennungsraten führen. Bei der aktuellen Erkennungsrate von bereits $91 \%$ brauchen wir aber darauf nicht mehr zu warten: Die automatische Pollenmessung ist schon jetzt einsatzbereit.

\section{Prof. Dr. Jeroen Buters}

ZAUM - Zentrum Allergie und Umwelt

Helmholtzzentrum München/Technische Universität München Biedersteiner Straße 29

80802 München

E-Mail: buters@tum.de

\section{Literatur}

1. Gilles S, Blume C, Wimmer M, Damialis A, Meulenbroek L, Gokkaya M et al. Pollen exposure weakens innate defense against respiratory viruses. Allergy 2020;75:576-87

2. Buters JTM, Antunes C, Galveias A, Bergmann KC, Thibaudon M, Galan $C$ et al. Pollen and spore monitoring in the world. Clin Transl Allergy 2018:8:9-13

3. Oteros J, Sofiev M, Smith M, Damialis T, Prank M, Werchan M et al. Building an automatic Pollen Monitoring Network [ePIN]: Selection of optimal stations by clustering pollen zones. Science Tot Environ 2019; 688:1263-74

4. Oteros-Moreno J, Pusch G, Weichenmeier I, Heimann U, Moeler R, Traidl-Hoffmann $\mathrm{C}$ et al. Automatic and on-line pollen monitoring. Int Arch Allergy Clin Immunol 2015;167:158-66

5. Oteros J, Weber A, Kutzora S, Rojo J, Heinze S, Herr C et al. Validation of an operational automatic pollen monitoring network based on image recognition in central Europe. Environ Res 2020; in review

6. Smith M, Oteros J, Schmidt-Weber C, Buters J. Short Communication: an abbreviated method for the Quality Control of pollen counters. Grana 2019; in Press

7. Oteros J, Bergmann K, Menzel A, Damialis A, Traidl-Hoffmann C, Schmidt-Weber $C$ et al. Spatial interpolation of current airborne pollen concentrations where no monitoring exists. Atmos Environ 2019;199: 435-42

8. Sauliene I, Sukiene L, Daunys G, Valiulis G, Vaitkevicius L, Matavulj P. Automatic pollen recognition with the Rapid-E particle counter: the first-level procedure, experience and next steps. Atmos Meas Tech 2019:12:3435-52

9. Crouzy B, Stella M, Konzelmann T, Calpini B, Clot B. All-optical automatic pollen identification: Towards an operational system. Atmos Environ 2016;140:202-12 